\title{
Intravenously transplanted bone marrow stromal cells promote recovery of lower urinary tract function in rats with complete spinal cord injury
}

\author{
$\mathrm{Y} \mathrm{Hu}^{1,2}, \mathrm{LM} \mathrm{Liao}^{1}, \mathrm{YH} \mathrm{Ju}{ }^{1}, \mathrm{G} \mathrm{Fu}{ }^{1}, \mathrm{HY} \mathrm{Zhang}^{3}$ and $\mathrm{HX} \mathrm{Wu^{2 }}$
}

Objectives: There is increasing evidence that intravenously injected neural progenitor cells promote recovery of bladder function in rodents, following contusive spinal cord injury through migrating into the injured spinal cord tissue and differentiating into central nervous system cells. The present study was aimed to clarify whether intravenously transplanted bone marrow stromal cells (BMSCs) could improve lower urinary tract (LUT) function in rats with spinal cord transection (SCT).

Methods: A total of 22 rats underwent experimentation in three groups, including group 1 -sham operation, group 2 (BMSC)—SCT plus BrdU (5-bromo-2'-deoxyuridine) labeled BMSCs transplantation at day 9 after SCT, group 3-SCT control. All rats were investigated urodynamically on day 28 after transplantation.

Results: BMSCs identified by BrdU immunohistochemistry survived in the injured spinal cord and lumbar level 3-4 ( $\mathrm{L}_{3-4}$ ). Voiding pressure, episodes of non-voiding contractions and residual urine volumes were significantly decreased in BMSC rats, compared with the controls. Bladder capacity was similar in both groups. In four out of eight BMSC rats and one out of seven controls, the tonic and bursting external urethral sphincter electromyographic activity were detected during cystometry. Silent periods during bursting were shorter and activity periods were longer in BMSC rats compared with sham rats.

Conclusion: Intravenously transplanted BMSCs survived in the $L_{3-4}$ and had beneficial effects on the recovery of LUT function in the rats after SCT.

Spinal Cord (2012) 50, 202-207; doi:10.1038/sc.2011.128; published online 29 November 2011

Keywords: spinal cord injury; lower urinary tract; mesenchymal stem cells; injections; intravenous; rats

\section{INTRODUCTION}

The main functions of the lower urinary tract (LUT) to store and periodically release urine are dependent upon neural circuits located in the brain, spinal cord and peripheral ganglia. ${ }^{1,2}$ Spinal cord injury (SCI) above the lumbosacral level interrupts this coordination between the bladder and the striated sphincter, leading to non-voiding contractions (NVCs) and detrusor-sphincter dyssynergia, which impedes voiding and leads to large residual urine volume (RUV). ${ }^{3}$ In the rats, micturition-associated bursting external urethral sphincter (EUS) activity, characterized by alternating bursts of EUS activity and relaxation (silent periods (SP)), is lost after SCI and has been reported to be replaced by tonic, dyssynergic activity. 4

As the mature central nervous system cannot generate new neurons and glial cells, bladder functional recovery is limited following SCI. However, recent studies suggest that transplanted neural progenitor cells promote recovery of the bladder function through regeneration of the injury site. ${ }^{5-8}$ In most of these studies, stem cells have been injected into the lesion directly with a needle, ${ }^{5,7,8}$ carrying the risk of further injury to the spinal cord. Otherwise, these studies did not examine the changes of EUS activity in the spinal injured rats after cells transplantation.
The present study was designed to demonstrate an improvement for LUT function in rats with spinal cord transection (SCT) by intravenous injection of bone marrow stromal cells (BMSCs).

\section{MATERIALS AND METHODS}

Animals

We used adult male Sprague-Dawley rats (PLA Academy of Military Science, Beijing, China) weighing between 200 and $250 \mathrm{gm}$ in this study. A total of 16 rats received SCT and intravenous transplantation of cell culture medium (control, $n=8$ ) or BMSCs (BMSC, $n=8$ ) at day 9 after SCT. Six laminectomyonly rats served as age-matched controls with an intact spinal cord (sham, $n=6)$. All the experiments were performed in compliance with relevant laws and institutional guidelines.

\section{Cell culture}

BMSCs obtained from donor male Sprague-Dawley rats (3-4 weeks old) were incubated in HyQ MEM Alpha Modification (HyClone, Logan, UT, USA) with $10 \%$ fetal bovine serum (Gibco, Grand Island, NY, USA) and 1\% antibiotics (penicillin-streptomycin; Gibco), and maintained at $37^{\circ} \mathrm{C}$ in $5 \% \mathrm{CO}_{2} / 95 \%$ ambient mixed air. For injection to animals, BMSCs were labeled with 5bromo-2'-deoxyuridine (BrdU) $\left(3 \mathrm{gg} \mathrm{ml}^{-1}\right.$, Sigma-Aldrich, St Louis, MO, USA) in vitro for 3 days. Passage 3-5 BMSCs were used.

${ }^{1}$ Department of Urology, China Rehabilitation Research Center, Rehabilitation College of Capital Medical University, Beijing, China; ${ }^{2}$ Department of Urology, Jinhua Municipal Central Hospital, Jinhua, Zhejiang, China and ${ }^{3}$ Basic Medicine College of Capital Medical University, Beijing, China

Correspondence: Professor LM Liao, Departmernt of Urology, China Rehabilitation Research Center, Rehabilitation College of Capital Medical University, No.10 Jiaomen Beilu, Fengtai District, Beijing 100068, China.

E-mail: Imlia064@yahoo.com.cn

Received 26 November 2010; revised 6 October 2011; accepted 9 October 2011; published online 29 November 2011 


\section{SCI and cell transplantation}

Rats were anesthetized with chloral hydrate $\left(360 \mathrm{mg} \mathrm{kg}^{-1}\right.$, intraperitoneally). After a T8-T9 laminectomy, the dura matter and spinal cord were cut with a scalpel blade and a section of spinal cord $(2 \mathrm{~mm})$ was removed. Gelfoam was placed between the severed ends of the spinal cord. The overlying muscle and skin were sutured. The bladder was expressed manually every $6-8 \mathrm{~h}$ until automatic micturition developed.

On day 9 after SCT, BMSCs $\left(3 \times 10^{6}\right.$ cells in $300 \mu$ l culture medium) were injected with a $1 \mathrm{ml}$ syringe into the tail vein of SCT rats. The control rats received intravenous injection of $300 \mu \mathrm{l}$ culture medium. No immunosuppression was performed during the investigations to prevent any confusion.

\section{Urodynamic assessment of LUT function}

Urodynamic tests were performed on day 28 after cell transplantation. Following urethane anesthesia $\left(1.2 \mathrm{~g} \mathrm{~kg}^{-1}\right.$, subcutaneously in sham rats and $0.8 \mathrm{~g} \mathrm{~kg}^{-1}$, subcutaneously in SCT rats), ${ }^{9}$ the urinary bladder was exposed via a middle abdominal incision and the rostral half of pubic symphysis was removed to expose the EUS. Two fine insulated silver needle electrodes $(0.3 \mathrm{~mm}$ diameter $)$ with exposed tips were inserted into lateral sides of the midurethra. A 20 gauge intravenous cannula was inserted through the bladder apex into the lumen for physiological saline infusion and bladder pressure measurements. ${ }^{10}$ The intravenous cannula was in turn connected via a 3-way stopcock to an infusion pump and a pressure transducer (TME, Chengdu, China). The electromyogram (EMG) activity was preamplified, filtered through a $1 \mathrm{kHz}$ filter, sampled at $5 \mathrm{kHz}$ and acquired simultaneously with the intravesical pressure.

Urodynamic tests usually began at 3-4h after the induction of anesthesia. After the bladder was emptied, cystometry was performed at an infusion rate of $0.1 \mathrm{ml} \mathrm{min}^{-1}$ with saline at room temperature. Three micturition cycles were collected after $30 \mathrm{~min}$ of saline infusion. The averages of the voiding pressure, RUV, bladder capacity and the number of NVCs per micturition episode were measured. A NVC was defined as a rhythmic intravesical pressure increase $>5 \mathrm{~mm} \mathrm{Hg}$ from baseline without a release of fluid from the urethra., ${ }^{5,6}$ At the time of voiding, saline infusion was stopped and voided saline was collected to determine voided volume. Then, residual saline was first withdrawn through the intravesical cannula and then the bladder was then expressed manually by applying pressure on the bladder wall to measure RUV. Bladder capacity was calculated as voided volume plus RUV. Voiding efficacy was estimated by the formula: (voided volume/bladder capacity) $\times 100$.

\section{Histology and immunohistochemistry}

After cystometry the animals were anesthetized with chloral hydrate (360 $\mathrm{mg} \mathrm{kg}^{-1}$, intraperitoneally) and killed. The entire spinal columns of rats were fixed by transcardial perfusion with saline, followed by perfusion and immersion in $4 \%$ paraformaldehyde. The spinal column was removed after which the entire spinal cord was dissected. Segments $40 \mathrm{~mm}$ rostral and caudal to the site of injury were paraffin embedded as well as lumbar level 3-4 $\left(\mathrm{L}_{3-4}\right)$, as this neurons in $\mathrm{L}_{3-4}$ were involved in the generation of EUS bursting activity during voiding. ${ }^{11}$ A series of adjacent $6 \mu \mathrm{m}$ coronal or longitudinal sections were cut and a section of each block of the spinal cord was stain with hematoxylin/eosin (H/E). Immunohistochemistry for BrdU was performed on serial sections using the avidin-biotin complex method.

\section{Statistical analysis}

Urodynamic data were analyzed using one-way ANOVA comparing groups. Post-hoc analysis was performed using Student-Newman-Keuls tests for continuous data or Fisher's exact test for categorical data. Significance levels were set to 0.05 for all comparisions.

\section{RESULTS}

\section{Histology}

Figure 1 shows three horizontal sections through the center of the whole spinal cord lesion. Obvious discontinuity of the spinal cord was evidence of SCT, which used the gelfoam for disconnecting almost all gray and white matter (Figure 1a). The ends of lesion area included cyst formations (star, Figure 1c), scarring (open block arrow, Figure 1c) and zones of microcysts (black arrows, Figures $1 \mathrm{~b}$ and c), where all are filled with gliosis and macrophages.

Figure 2 Immunohistochemistry confirmed the survival of transplanted BMSCs on day 28 after transplantation. BrdU immunoreactive cells were found in the lesioned spinal cord and $\mathrm{L}_{3-4}$ (Figures $2 \mathrm{a}$ and c). Many transplanted cells seemed to migrate into the dorsal spinal cord, especially in the white matter and dorsal gray commissure around the central canal (Figures $2 \mathrm{~b}$ and $\mathrm{d}$ ).

\section{Anesthetized cystometry}

Figure 3 shows typical cystometry charts for sham, control and BMSC rats. As saline was infused into the bladder, rhythmic intravesical a
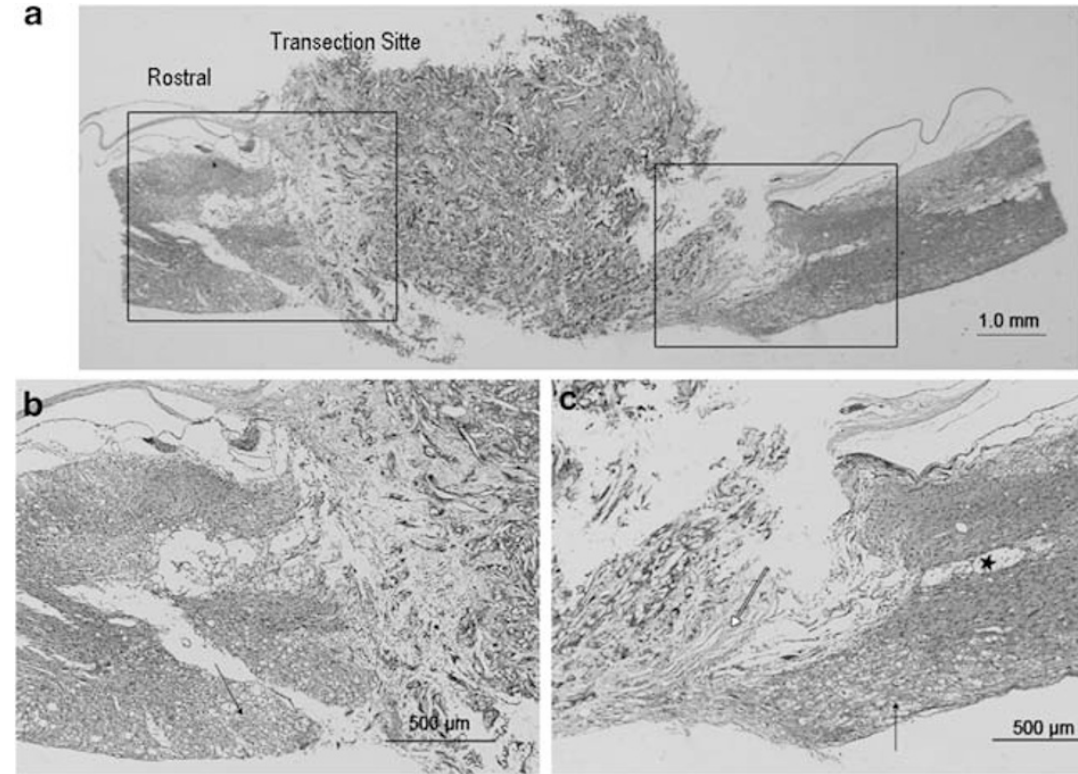

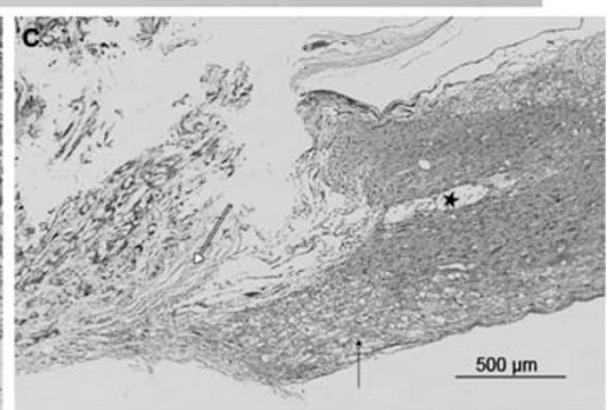

Figure 1 The representative horizontal sections, stained with $\mathrm{H} / \mathrm{E}$, taken through the spinal cord of a rat with SCT. Gelfoam can be seen at the transection site. Note the absence of $\mathrm{H} / \mathrm{E}$-stained gray and white matter crossing the transection site (a). The black arrows indicate some regions of microcysts, an open block arrow indicate scarring and an asterisk indicates cystic cavities (b, $\mathbf{c})$. 
pressure waves appeared before voiding in control and BMSC group, but not in sham rats. These pressure waves, termed NVCs, were not associated with saline release from the urethra. As detailed in Table 1, there were fewer NVCs in BMSC rats, compared with the controls. Filling duration was significantly longer in SCT rats than sham rats, indicating that bladder capacity of spinal injured rats was larger than sham rats.

\section{Urodynamic data}

As detailed in Table 1, because one of controls died after the surgery, cystometry was done in six sham, seven control and eight BMSC rats. ANOVA revealed significant differences among the three groups for all parameters. Post-hoc analysis showed differences between control and sham rats for voiding pressure $(P<0.01)$, number of NVCs $(P<0.01)$, the bladder capacity $(P<0.01)$, RUV $(P<0.01)$ and voiding efficiency $(P<0.01)$. BMSC rats had lower voiding pressure $(P<0.01)$, fewer episodes of NVCs $(P<0.01)$, less RUV $(P<0.05)$ and better voiding efficiency $(P<0.01)$ than controls. Although cystometry parameters in the BMSC group were improved compared with controls, they remained significantly different from sham group in voiding pressure $(P<0.01)$, bladder capacity $(P<0.01)$, RUV $(P<0.05)$ and voiding efficiency $(P<0.01)$.

\section{EUS EMG}

Figure 3 shows a bladder contraction during voiding is accompanied by large-amplitude EUS EMG activity (bursting activity) in all sham rats. NVCs were accompanied by an increasing EUS EMG activity (tonic activity) during bladder filling in SCT rats. Figure 4 shows typical EUS EMG for sham, control and BMSC rats during voiding. In all, 2 types of EUS EMG activity were detected during voiding in all sham and half of BMSC rats. In these rats, the EUS EMG activity markedly increased during bladder contractions (Figure 4a) and
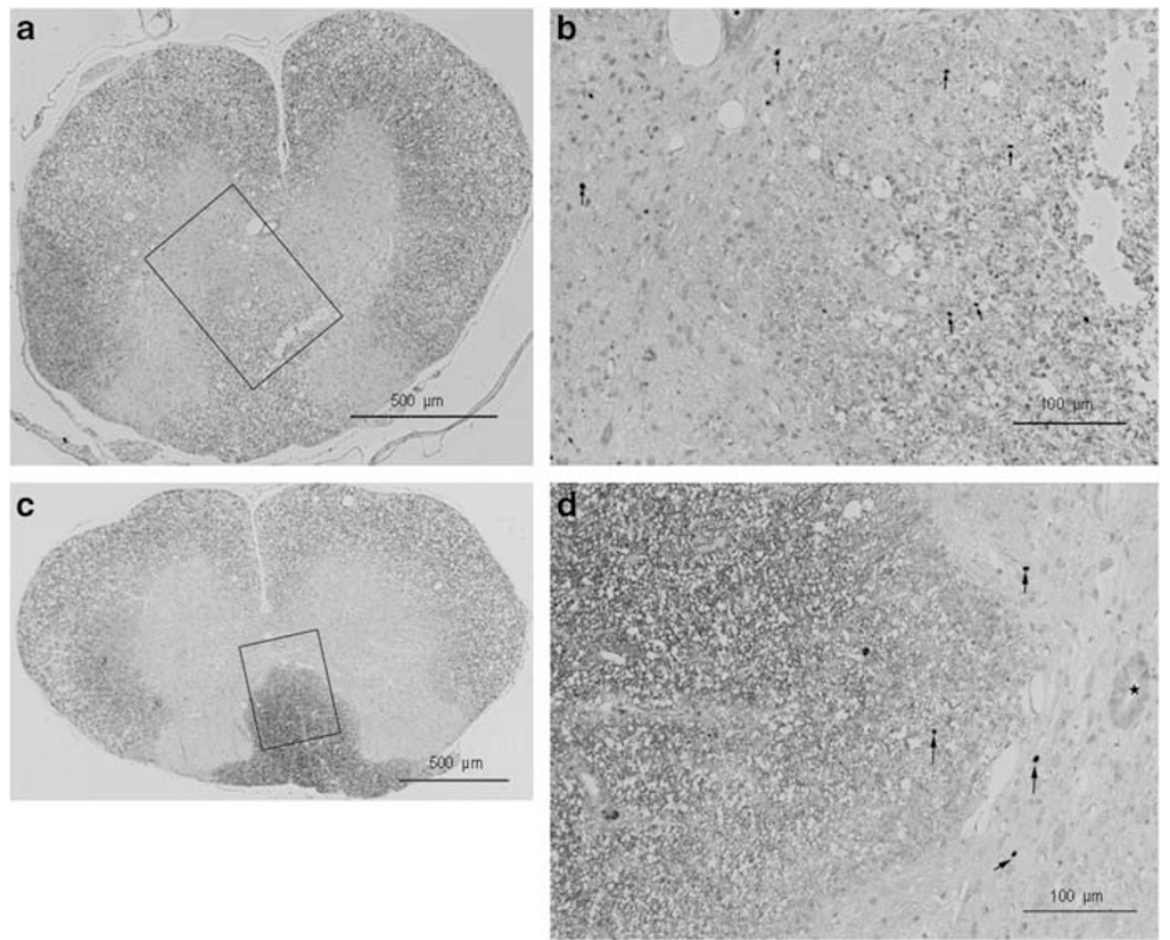

Figure 2 Immunohistology of transplanted BrdU reactive cells in spinal cord. Black dots represent immunoreactivity to BrdU. (a) On injured spinal cord coronal section immunohistochemistry confirmed transplanted cell survival. Reduced from $\times 40$. (b) BrdU immunoreactive cells (black nuclei) were found in the dorsal spinal cord, especially in the white matter around the central canal (asterisk). Reduced from $\times 200$. (c) On $L_{3-4}$ coronal section immunohistochemistry confirmed transplanted cell survival. Reduced from $\times 40$. (d) BrdU reactive cells (black nuclei) show that some transplanted cells seemed to migrate into the dorsal spinal cord $\left(L_{3-4}\right)$. Reduced from $\times 200$.

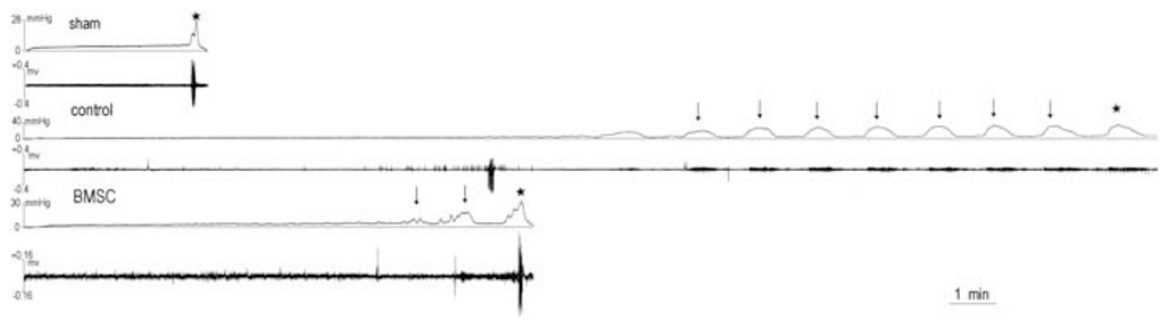

Figure 3 Representative cystometry charts of sham, control and BMSC shows that during bladder saline infusion NVC $(\downarrow)$ are frequent before voiding in control compared with BMSC, and are not seen in sham. NVC was not associated with urethral saline release. Saline leakage started (asterisk) at the full bladder. Note: higher voiding pressure, more numbers of NVC and longer duration of the bladder filling before leakage in control than in BMSC. Horizontal calibration: time in min; vertical calibration: intravesical pressure in $\mathrm{mm} \mathrm{Hg}$, EUS EMG in mv. 
Table 1 Cystometry parameters and the bladder weight

\begin{tabular}{lccc}
\hline Parameters & Sham & OP-control & BMSCs \\
\hline Voiding pressure (mm Hg) & $26.35 \pm 4.18$ & $40.74 \pm 3.97^{\mathrm{aa}}$ & $33.82 \pm 3.79^{\mathrm{bb}, \mathrm{cc}}$ \\
Residual urine $(\mathrm{ml})$ & $0.10 \pm 0.08$ & $1.53 \pm 0.68^{\mathrm{aa}}$ & $0.89 \pm 0.17^{\mathrm{b}, \mathrm{c}}$ \\
Bladder capacity (ml) & $0.35 \pm 0.14$ & $1.57 \pm 0.68^{\mathrm{aa}}$ & $1.20 \pm 0.22^{\mathrm{bb}}$ \\
Voiding efficiency (\%) & $70.83 \pm 10.10$ & $2.78 \pm 1.46^{\mathrm{aa}}$ & $26.25 \pm 6.93^{\mathrm{bb}, \mathrm{cc}}$ \\
NVC (number of episodes/micturition) & 0 & $9.67 \pm 3.83^{\mathrm{aa}}$ & $2.13 \pm 1.13^{\mathrm{cc}}$ \\
\hline
\end{tabular}

aa $P<0.01$, sham versus control.

${ }^{\mathrm{b}} P<0.05, \mathrm{bb} P<0.01$, sham versus BMSCs.

${ }^{c} P<0.05$, ${ }^{\text {cc }} P<0.01$, control versus BMSCs.
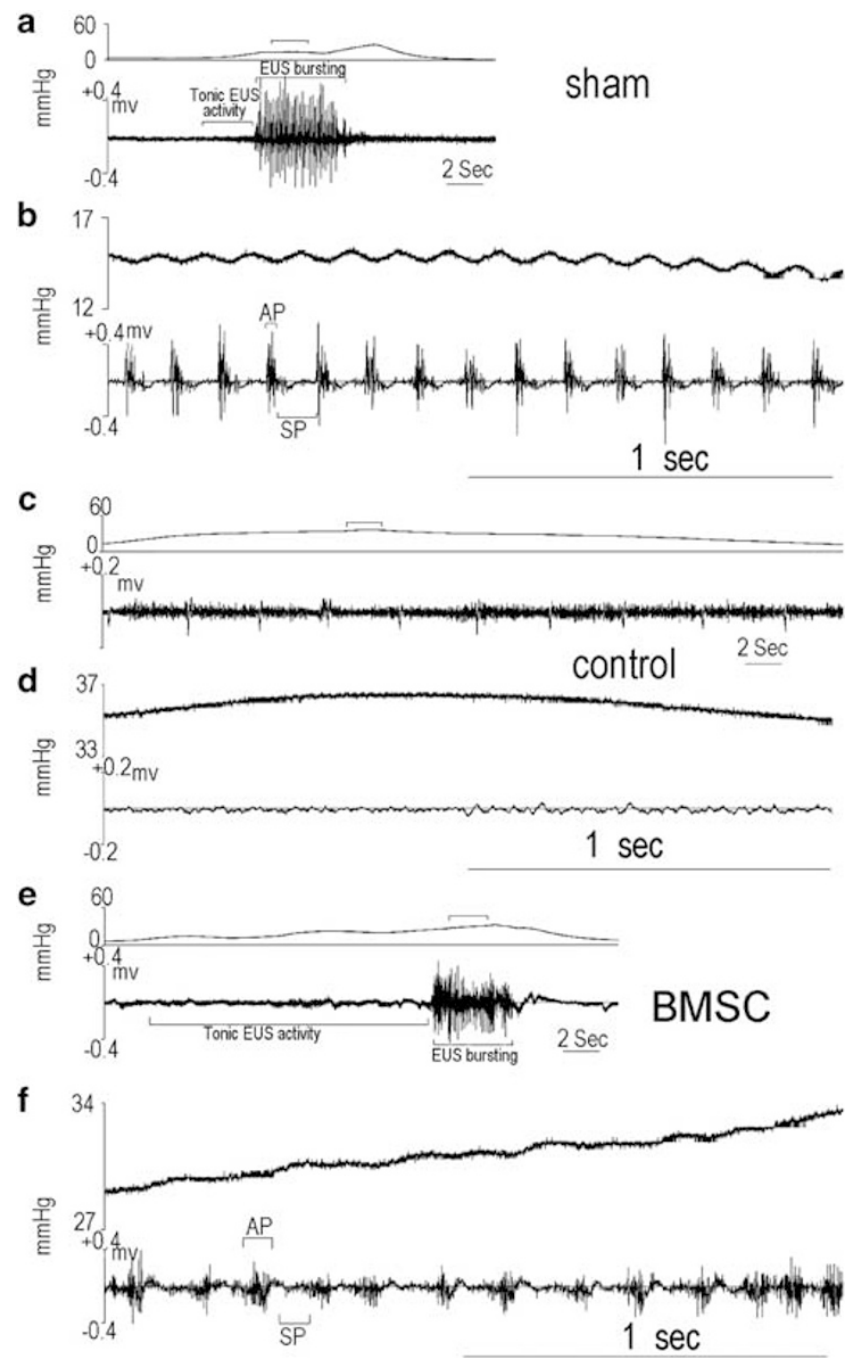

consisted of an initial period of tonic activity followed by a bursting pattern of activity characterized by clusters of high-frequency spikes (active period (AP)) separated by periods of quiescence (SP) (Figure $4 \mathrm{~b}$ ). In sham rats, the average duration of the bursting period was $5.0 \pm 2.76 \mathrm{~s}$ and consisted of the SP that was obviously longer $(131.78 \pm 17.07 \mathrm{~ms})$ than the AP $(38.10 \pm 9.63 \mathrm{~ms})$ (Table 2). In controls, only $14.29 \%$ rats $(P<0.01)$ demonstrated bursting activity, significantly fewer than the sham group. Bursting activity was detected in $50 \%$ of BMSCs rats, which was more than controls $(P<0.05)$. However, in the BMSC group, the AP was significantly longer
Figure 4 The bladder (top traces) and EUS EMG activity (bottom traces) recorded during the period of voiding in an anesthetized sham, control and BMSC rats. The bracket in (a) indicates the recording period in (b), the bracket in (c) indicates the recording period in (d) and the bracket in (e) indicates the recording period in (f) at faster time scales. (a) Tonic EUS EMG activity precedes the large rise in intravesical pressure and shifts to a bursting pattern at the peak of the bladder contraction before the onset of voiding, with the decline in intravesical pressure during EUS EMG bursting. (b) Recordings are shown at very fast time scale showing individual bursts composed of AP (brackets) and SP (brackets) and the small fluctuations in intravesical pressure accompanying each burst. (c) Only tonic EUS EMG activity appears in control during the leakage of saline, which reflects detrusor-sphincter dyssynergia. (d) Recordings in (c) shown at very fast time scale, showing that there are no active, and SP and fewer small waves in intravesical pressure. (e) Longer tonic EUS activity and shorter EUS bursting appears in the BMSC rats during the period of saline leakage. (f) Recordings in (e) shown at very fast time scale, showing there are longer AP and shorter $\mathrm{SP}$, and the small fluctuations in intravesical pressure accompanying each burst. Horizontal calibration: times in s; vertical calibration: intravesical pressure in $\mathrm{mm} \mathrm{Hg}$, EUS EGM in $\mathrm{mv}$.

$(P<0.01)$, and the SP $(P<0.01)$ and SP/AP ratio $(P<0.05)$ were significantly less than sham rats.

\section{DISCUSSION}

In spinal cord-intact rats, when the bladder is filling, the $\mathrm{L}_{6}-\mathrm{S}_{1}$ spinal cord accepts afferent input from bladder mechanoreceptors to generate tonic EUS activity mediated a spinal reflex pathway. Although the bladder is voiding, pontine micturition center projects to the lumbosacral micturition center to generate bladder contractions and to $\mathrm{L}_{3-4}$ bursting center to generate EUS bursting. ${ }^{11}$ SCI above the lumbosacral level interrupts the coordination between the bladder and EUS, inducing the appearance of reflex micturition and NVCs, mediated by the reemergence of spinal reflex pathways via C-fiber bladder afferents that are not essential for normal voiding. ${ }^{12}$ However, the reflex micturition is inefficient because of persisting detrusorsphincter dyssynergia, which is induced by activity of bladder C-fiber afferents in deeply anesthetized SCI rats. ${ }^{9}$

In our study, we found significant recovery of bladder function in BMSC rats. First, we found an improvement (decrease) in the incidence of NVCs during bladder filling. It has been speculated that directly injected stem cells inhibit unmediated C-fiber bladder afferent sprouting and thereby decrease a C-fiber bladder-to-bladder spinal micturition reflex in SCI rats. ${ }^{6}$ However, it is different to a recent study on transplantation of neuronal-glial restricted precursors or neural cells to rats with traumatic SCI, ${ }^{8}$ suggesting that recovery of function mediated by parasympathetic systems may be more difficultly accomplished than that mediated by somatic pathways. ${ }^{5}$ Secondly, significant improvements were observed in voiding pressure, RUV and voiding efficiency during micturition, which were attribu- 
Table 2 EUS EMG

\begin{tabular}{lccc}
\hline Parameters & Sham & OP-control & BMSCs \\
& $\mathrm{n}=6$ & $\mathrm{n}=1$ & $\mathrm{n}=4$ \\
\hline Incident of EUS bursting (\%) & $100(6 / 6)$ & $14.29^{\mathrm{aa}}(1 / 7)$ & $50^{\mathrm{bb}, \mathrm{c}}(4 / 8)$ \\
Bursting period (s) & $5.0 \pm 2.76$ & 4 & $3.62 \pm 1.9$ \\
Tonic activity period (s) & $3.37 \pm 4.51$ & 10.4 & $7.24 \pm 3.12$ \\
AP (ms) & $38.10 \pm 9.63$ & 107.0 & $88.53 \pm 18.21^{\mathrm{bb}}$ \\
SP (ms) & $131.78 \pm 17.07$ & 32.5 & $69.0 \pm 13.75^{\mathrm{bb}}$ \\
SP/AP & $3.67 \pm 1.17$ & 0.3 & $0.78 \pm 0.09^{\mathrm{b}}$
\end{tabular}

Silent and AP denotes the average duration of quiescent and tonic EUS EMG activity during bursting period shown in Figure 4. Enumeration data of all groups is shown as mean \pm s.e., except when $n<2$, in which case individual values are reported. Enumeration data of groups with $n>3$ and incident rate were used for statistical comparisons.

aa $P<0.01$, sham versus control.

${ }^{\mathrm{b}} P<0.05$, bb $P<0.01$, sham versus BMSCs.

${ }^{c} P<0.05,{ }^{c c} P<0.01$, control versus BMSCs.

table to alleviating EUS dysfunctions. However, there was no decrease in the bladder capacity in BMSC group, compared with controls. Such an increase in the bladder capacity is thought to be caused by the bladder overdistention during the areflexic period before cells transplantation.

We also observed the recovery of the urethral function and coordination between the bladder and EUS. The EUS EMG was acquired simultaneously with the intravesical pressure. It was a new view of the recovery of the urethral function, comparing with the similar studies. ${ }^{5,78}$ This could clearly prove the recovery of bladdersphincter synergia in rats after BMSC transplantation. We analyzed the EUS EMG pattern to show that the EUS exhibited bursting activity which appeared to facilitate voiding in sham rats; whereas the EUS exhibited tonic activity which inhibited voiding in SCI rats. ${ }^{3}$ EUS bursting activity consists of SP, when the urethra is relaxed, permitting urine to be expelled, and AP, when the urethra is closed. ${ }^{9}$ It is thought to be necessary for bladder-sphincter synergia during voiding in rats. In half of the BMSC rats, we observed EUS bursting activity, longer SP and shorter AP during voiding under urethane anesthesia. These results were similar with the previous studies that EUS bursting activity was not detected in all SCI rats under anesthesia, ${ }^{9,13}$ because of the depressant effect of light urethane on voiding function. On the other hand, efficient voiding depends on the duration and number of urethral openings during voiding. As shown in Table 2, the SP was shorter in BMSC rats than in sham rats, whereas the AP was increased. Thus, although bursting occurs in BMSC rats, it is abnormal (that is, $78.75 \%$ shorter SP/AP), presumably contributing to the higher voiding pressure, larger RUV and less efficient voiding than the sham rats, because bursting activity is less robust in the BMSC rats than in rats with an intact neuraxis. ${ }^{9}$

In the current study, we used gelfoam for keeping two severed ends of spinal cord separate, which was confirmed by H/E staining of the injured spinal cord. There were no connections of the gray and white matter in BMSC rats, although BrdU immunoreactive cells were found rostrally and caudally from the lesion area. Chopp et al. ${ }^{14}$ described that the homing of intravenously infused mesenchymal stem cells to the sites of injury was reminiscent of inflammatory cells to the injured tissue, and similar mechanisms were considered for BMSCs. These BrdU-labeled BMSCs cannot promote partial sparing of descending modulatory pathways that course through the lateral and ventral funiculi to the lumbosacral cord in SCT rats, which is a reasonable hypothesis responsible for the bladder function improvement in rats with spinal cord contusion. ${ }^{6,7}$ So what is the actual mechanism for the LUT function recovery in BMSC rats? The discovery of BrdU-labeled
BMSCs in the dorsal gray commissure of $\mathrm{L}_{3-4}$ indicates that the reorganization of synaptic connections may be taken place, which is responsible for the formation of the new pathways of voiding reflex in BMSC rats. Marson ${ }^{15}$ and Lee et al. ${ }^{16}$ found that neurons in the dorsal gray commissure activated with the pudendal or pelvic nerve stimulation might integrate afferent signal neurons within multiple spinal segments and relay important sensory information to the brain in spinally intact rats. On the other hand, Dolber et al. ${ }^{17}$ and Chang et al. ${ }^{11}$ proposed a hypothetical framework for the coordinated bladder contraction and bursting EUS relaxation in the SCI rats including a capsaicin-insensitive afferent input to a switch, which directly or indirectly controls excitation of the parasympathetic preganlionic neurons and relaxation of the Onuf's nucleus motor neurons. In our study, BMSCs in the dorsal gray commissure, presenting proximal to both the sacral parasympathetic preganglionic neurons and the spinal EUS pattern generator, may promote the formation of the switch to coordinate bladder contraction and EUS relaxation by expressing neuronal and astroglial markers ${ }^{18}$ or secreting neurotrophic factor and cytokine. ${ }^{19}$

In the current study, we did not examine how these BMSCs differentiated after transplantation. Clearly, this is a limitation of the study. In addition, a recent study showed that BMSCs were homed into partial bladder outlet obstruction bladder and differentiated into the several components of the bladder tissue, contributing to the regeneration of the partial bladder outlet obstruction bladder (2011 AUA annual meeting abstract 429). These findings indicate that there may be other mechanisms for the improved bladder function in BMSC rats, on which we will do more work in our further research.

\section{CONCLUSIONS}

Intravenously transplanted BMSCs survived in the $\mathrm{L}_{3-4}$ at least 4 weeks. Some improvements in LUT function were observed following BMSCs-transplantation in SCT rats. Although the current study is preliminary, intravenous transplantation of BMSCs may have some potentiality for treating LUT dysfunction after SCI in humans.

\section{CONFLICT OF INTEREST}

The authors declare no conflict of interest.

\section{ACKNOWLEDGEMENTS}

This work was supported by a grant (No.2008BAI50B06) from the China National Key Technology R\&D Program (Prof. Limin Liao).

1 Morrison J, Steers WD, Brading A, Blok B, Fry C, de Groat WC et al. Neurophysiology and neuropharmacology. In: Abrams P, Cardozo L, Khoury S, Wein A (eds). Incontinence. Health Publications Ltd: Jersey, 2002, pp 83-164.

2 de Groat WC, Yoshimura N. Mechanisms underlying the recovery of lower urinary tract function following spinal cord injury. Prog Brain Res 2006; 152: 59-84.

3 Kruse MN, Belton AL, de Groat WC. Changes in bladder and external urethral sphincter function after spinal cord injury in the rat. Am J Physiol 1993; 264: 1157-1163.

4 Kruse MN, Bennett B, de Groat WC. Effect of urinary diversion on the recovery of micturition reflexes after spinal cord injury in the rat. J Urol 1994; 151: 1088-1091.

5 Mitsui TA, Kakizaki HI, Tanaka HI, Shibata TA, Matsuoka IC, Koyanagi TO. Immortalized neural stem cells transplanted into the injured spinal cord promote recovery of voiding function in the rat. J Urol 2003; 170: 1421-1425.

6 Mitsui T, Fischer I, Shumsky JS, Murray M. Transplants of fibroblasts expressing BDNF and NT-3 promote recovery of bladder and hindlimb function following spinal contusion injury in rats. Exp Neurol 2005; 194: 410-431.

7 Mitsui T, Shumsky JS, Lepore AC, Murray M, Fischer I. Transplantation of neuronal and glial restricted precursors into contused spinal cord improves bladder and motor functions, decreases thermal hypersensitivity, and modifies intraspinal circuitry. J Neurosci 2005; 25: 9624-9636. 
8 Temeltas G, Dagci T, Kurt F, Evren V, Tuglu I. Bladder function recovery in rats with traumatic spinal cord injury after transplantation of neuronal-glial restricted precursors or bone marrow stromal cells. J Urol 2009; 181: 2774-2779.

9 Cheng $\mathrm{CL}$, de Groat WC. The role of capsaicin-sensitive afferent fibers in the lower urinary tract dysfunction induced by chronic spinal cord injury in rats. Exp Neurol 2004; 187: 445-454.

10 Maggi CA, Santicioli P, Meli A. The nonstop transvesical cystometrogram in urethaneanesthetized rats: a simple procedure for quantitative studies on the various phases of urinary bladder voiding cycle. J Pharmacol Methods 1986; 15: 157-167.

11 Chang HY, Cheng CL, Chen JJ, de GW. Serotonergic drugs and spinal cord transections indicate that different spinal circuits are involved in external urethral sphincter activity in rats. Am J Physiol Renal Physiol 2007; 292: 1044-1053.

12 Yoshimura N. Bladder afferent pathway and spinal cord injury: possible mechanisms inducing hyperreflexia of the urinary bladder. Prog Neurobiol 1999; 57: 583-606.

13 Leung PY, Johnson CS, Wrathall JR. Comparison of the effects of complete and incomplete spinal cord injury on lower urinary tract function as evaluated in unanesthetized rats. Exp Neurol 2007; 208: 80-91.
14 Chopp M, Li Y. Treatment of neural injury with marrow stromal cells. Lancet Neurol 2002; 1: 92-100.

15 Marson L. Central nervous system neurons identified after injection of pseudorabies virus into the rat clitoris. Neurosci Lett 1995; 190: 41-44.

16 Lee JW, Erskine MS. Pseudorabies virus tracing of neural pathways between the uterine cervix and CNS: effects of survival time, estrogen treatment, rhizotomy, and pelvic nerve transection. J Comp Neurol 2000; 418: 484-503.

17 Dolber PC, Gu B, Zhang X, Fraser MO, Thor KB, Reiter JP. Activation of the external urethral sphincter central pattern generator by a $5-\mathrm{HT}(1 \mathrm{~A})$ receptor agonist in rats with chronic spinal cord injury. Am J Physiol Regul Integr Comp Physiol 2007; 292. 1699-1706.

18 Zurita M, Vaquero J. Functional recovery in chronic paraplegia after bone marrow stromal cells transplantation. Neuroreport 2004; 15: 1105-1108.

19 Himes BT, Neuhuber B, Coleman C, Kushner R, Swanger SA, Kopen GC, et al. Recovery of function following grafting of human bone marrow-derived stromal cells into the injured spinal cord. Neurorehabil Neural Repair 2006; 20: 278-296. 